
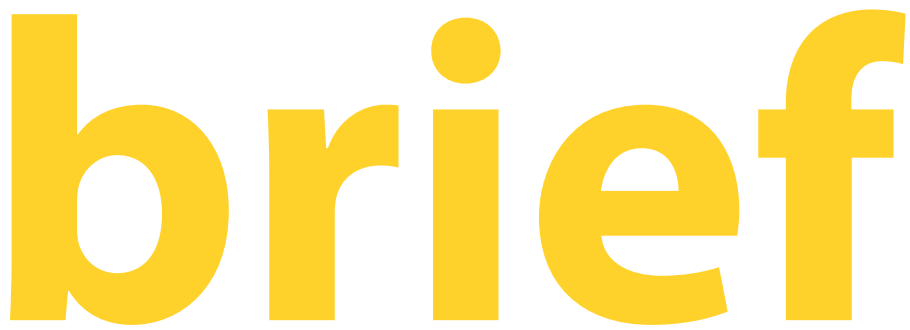

Les briefs du CIFOR vous proposent des informations concises, exactes et d'ordre scientifique sur des sujets d'actualités de la recherche forestière.

\section{Le Paysage Virunga}

Chrislain Eric Kenfack

\section{Géographie et climat}

Avec une superficie totale de 15155 km², le paysage Virunga, du Nord au Sud, s'étend le long des frontières de la République démocratique du Congo, du Rwanda et de l'Ouganda. $56 \%$ du paysage est couvert par les aires protégées, notamment: le parc national des Virunga (772 $700 \mathrm{ha}$ ), le parc national des Volcans (16 $000 \mathrm{ha})$, une bande de $10 \mathrm{~km}$ de large le long de ces parcs, la réserve de Sarambwe (900 ha) et le domaine de chasse de Rutshuru (64 200 ha) (Etat des forêts 2006).

Le paysage est constitué de trois grandes écorégions terrestres : l'écorégion de l'afro-alpine stérile de RuwenzoriVirunga, l'écorégion de la forêt afro-montagneuse de la fissure Albertine, et l'écorégion de la mosaïque forêt-savane du lac Victoria. On y retrouve également les écorégions aquatiques des montagnes de la fissure Albertine, des lacs Kivu, Edouard, George, Burera, Ruhondo et Victoria, et les trois grands massifs montagneux, Mitumba, Ruwenzori et Virunga.

Le régime climatique du paysage est de type bimodal, avec deux saisons des pluies (septembre-décembre et mars-mai), et deux saisons sèches (janvier et juillet). La température moyenne annuelle est comprise entre $20^{\circ}$ et $23^{\circ} \mathrm{C}$. Le paysage est situé à une altitude comprise entre 680 et 5119 mètres.

\section{Populations}

L'on retrouve près de 2 millions d'habitants dans les zones urbaines, et presque autant dans les zones rurales. La densité moyenne de la populaion est de 200 à 300 habitants $/ \mathrm{km}^{2}$, et atteint par endroit 600 habitants $/ \mathrm{km}^{2}$ (Wengamulay 2011).

Les principaux groupes ethniques sont : les Nande, les Hutu, les Tutsi, les Hunde et les Kumu possédant des terres ancestrales. On retrouve également une mosaïque d'ethnies présente dans les grandes agglomérations.

La principale activité du paysage est l'agriculture de subsistance. Cependant, une minorité d'habitants pratique l'élevage, la pêche et la chasse, aussi bien pour des besoins de consommation que pour des besoins commerciaux.

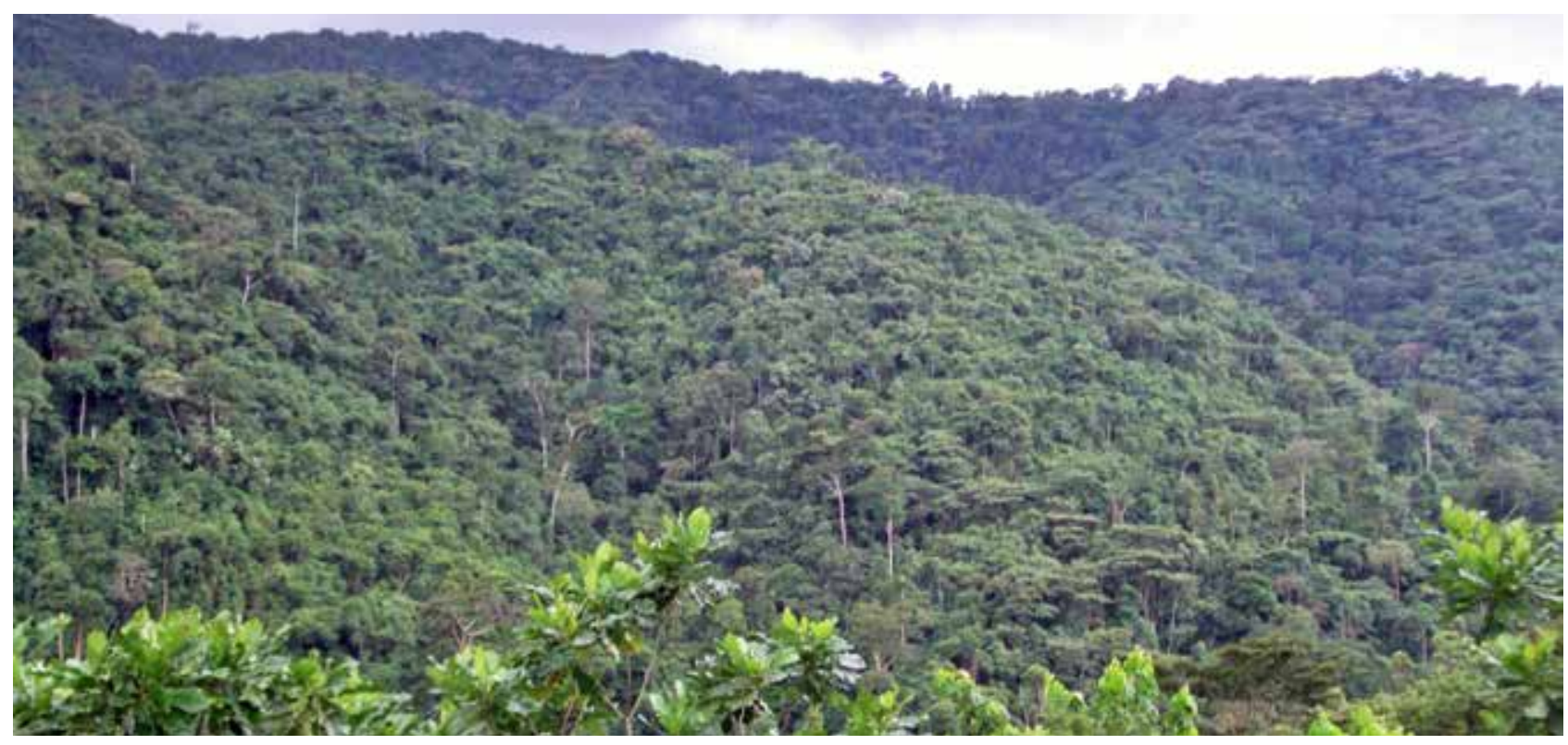




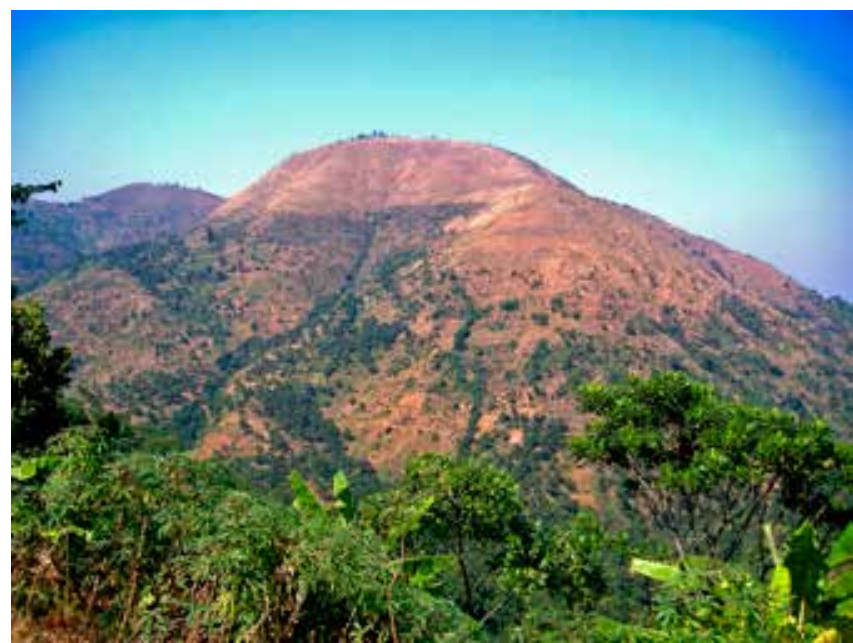

Vue typique du paysage Virunga montrant au passage les difficultés d'accès aux terres (Auteur: Anne Marie Tiani)

\section{Couvert forestier}

Selon les statistiques datant de 2005, le couvert forestier est estimé à 3143 km² (Etat des forêts 2008).

\section{Végétation}

Le paysage Virunga est essentiellement constitué de forêts denses et humides. Toutefois, l'on y retrouve de la savane et des mosaïques forêts-cultures. A ce titre, il faut noter que la partie Nord du paysage (principalement dans les zones de Ruwenzori et Semliki), et la partie Sud (principalement dans les zones de Mikeno, Karisimbi, Gahinga, Sabyinyo, Muhabura, Nyiragongo, Nyamuragira) sont boisées, alors que les autres zones sont essentiellement des savanes.

\section{Déforestation}

L'agriculture et l'exploitation du bois pour des besoins énergétiques et artisanaux sont les principaux moteurs de déforestation. En effet, en dehors des zones protégées, 80\% des terres sont utilisées entre autres pour l'agriculture de subsistance (maïs, pommes de terre, haricot, sorgho, etc.) et l'agriculture industrielle (café, thé, cacao, quinquina, etc.). Par ailleurs, le bois et le charbon de bois issus de l'exploitation artisanale des forêts constituent la principale source d'énergie des populations vivant dans le paysage.

Les superficies ayant été déboisées entre 2000 et 2005 sont aujourd'hui estimées à $136 \mathrm{~km}^{2}$, avec un taux annuel de déforestation évalué à 4,14 \% (Etat des forêts 2008).

En 2004, le parc national des Virunga a perdu 1500 ha de forêt, du fait des pressions exercées par les populations venues du Rwanda. Plus de $90 \%$ de la superficie dans le domaine de chasse de Rutshuru est totalement dégradée et transformée en champs de culture vivrière (Etat des forêts 2006).

\section{Biodiversité}

Caractérisé par une grande richesse et un endémisme exceptionnel, le paysage Virunga compte plus de 210 espèces de mammifères (nombre en diminution ces dernières décennies du fait de la surexploitation et des activités de braconnage), 706 espèces d'oiseaux, 109 espèces de reptiles et 78 espèces d'amphibiens. On y retrouve aussi 22 espèces de primates et 3 taxons de grands singes, notamment les gorilles de montagne (Gorilla beringei beringei), les gorilles de plaine de l'Est (Gorilla beringei graueri) et les chimpanzés de l'Est (Pan troglodytes schweinfurthi) (Etat des forêts 2008).

On y repère également une petite population d'okapis (Okapia johnstoni), principalement sur les rives de la rivière Semliki dans la forêt équatoriale non dégradée. Toutefois, cette population reste menacée par la chasse commerciale et la production du charbon de bois, tout comme le sont plusieurs variétés de poissons dans le lac Edouard, à cause de la surpêche pratiquée par les populations.

\section{Menaces pour la conservation de la biodiversité} Les principaux facteurs de perte de la biodiversité sont :

L’agriculture. L'activité agricole dans le paysage se pratique essentiellement à l'aide des méthodes archaïques, causant une production insuffisante à couvrir les besoins des populations. Pour augmenter la production agricole, les exploitations ont besoin d'utiliser plus d'étendue de terres, avec pour conséquence l'augmentation de la pression exercée sur les ressources naturelles, au point d'occasionner une perte d'environ 8\% de forêt.

De plus, la non diversification des activités et des sources de revenus pousse les populations à se tourner presqu'exclusivement vers l'exploitation des ressources forestières. Par exemple, les communautés sont dépendantes des espèces telles les bambous et les essences forestières pour des besoins agricoles et énergétiques. Dès lors, celles-ci sont en situation constante de stress, qui se manifeste à travers la baisse de la productivité et l'effondrement des stocks.

Le braconnage. L'une des plus grandes menaces demeure le braconnage intensif. II est pratiqué pour combler les besoins des populations en protéines animales d'une part, et d'autre part pour le trafic, spécialement des produits tels que les défenses d'éléphants, les canines d'hippopotames, et les bébés gorilles.

\section{Affectation des terres}

L'aménagement de l'affectation des terres en est encore à ses débuts, et il n'existe, à l'heure actuelle, aucune concession forestière. Toutefois, le paysage Virunga est essentiellement caractérisé par la présence de deux macro-zones :

- Les aires protégées : le parc national des Virunga (déclaré Patrimoine mondial depuis 1979), le parc national des volcans (réserve de biosphère), et le domaine des chasses de Rutshuru.

- Les zones de gestion communautaire de ressources naturelles (GCRN) : les zones de Djuma, Mwenda et Bwisha en RDC, et la zone de Kinigi-Burera au Rwanda. 


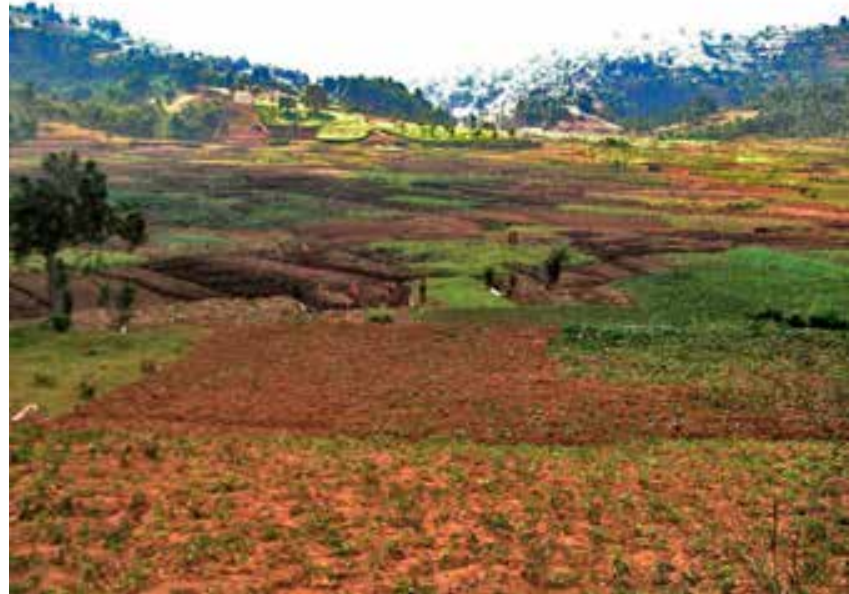

Modes d'utilisation des bas-fonds dans le paysage Virunga (Auteur : Anne Marie Tiani)

Les principales activités liées à l'affectation des terres (Etat des forêts 2008) sont :

- La conservation (55\%)

- L'agriculture (42\%)

- $\quad$ La pêche (3 \%)

On peut également noter l'exploitation minière artisanale qui, bien que n'étant pas très importante, occupe $5 \%$ des activités du paysage (Etat des forêts 2006).

\section{Partenaires institutionnels}

Comme principaux partenaires institutionnels que l'on retrouve dans le paysage Virunga, on peut citer:

- $\quad$ ICCN : Institut congolais pour la conservation de la nature

- $\quad$ RDB : Rwanda Development Board

- $\quad$ RNRA : Rwanda Natural Resources Authority

- $\quad$ AWF : African Wildlife Foundation

- UMD-CARPE : University of Maryland - Programme régional de l'Afrique centrale pour l'environnement.

- OSFAC : Observatoire satellital des forêts d'Afrique centrale

- FORAF : Forêts d'Afrique centrale

- UICN : Union internationale pour la conservation de la nature

- Tom Patterson

- US National Park Service

- $\quad \mathrm{FFI}$ : Fauna \& Flora International

- WWF : Fonds mondial pour la nature

- ZSL : Société zoologique de Londres

\section{Opportunités, adaptation et REDD+}

Certaines actions sont entreprises dans le paysage Virunga pour la préservation de la forêt et des ressources naturelles, du fait de leur importance dans l'adaptation et l'atténuation des changements climatiques. Notamment en termes de :

- Reboisement communautaire dans le paysage. Le paysage a fait l'objet de 600 ha de reboisement dans 4 territoires à savoir Masisi, Rutshuru, Beni et Lubero (Ko 2011). Cette activité, en plus du rétablissement progressif de la végétation, permet de cultiver le sens de l'appartenance et de la protection de la nature chez les populations vivant dans le paysage.

- Fabrication des briquettes combustibles en terre stabilisées. Cette activité permet de gagner 45 dollars américains par mois par habitant, améliorant ainsi la capacité d'adaptation à travers la génération des revenus et la réduction de la dégradation des sols.

- Aménagement des étangs piscicoles dans les zones humides. Les étangs permettent de ravitailler les populations en produits aquatiques. Ils permettent également de préserver la riche biodiversité aquatique du paysage, tout en renforçant la capacité d'adaptation des communautés par la diversification de leurs modes d'existence.

- $\quad$ Programme environnemental de Virunga. Supporté par WWF depuis 1987, c'est un programme à long terme qui vise la protection de l'écosystème de Virunga, à travers la formation et l'éducation environnementales des gestionnaires et des populations. Il a également pour objectif le développement économique et la création d'énergies alternatives dans le paysage et ses environs, facilitant ainsi la résilience climatique et la mise en œuvre des projets de conservation tels que la REDD+.

- Construction et maintenance des points d'adduction d'eau. Cette activité permet de fournir de l'eau aux populations et au bétail, à partir des rivières du paysage, réduisant alors le risque d'indisponibilité d'eau lié au climat.

- Subventions accordées aux communautés. Ces appuis (distribution d'intrants agro-pastoraux aux communautés regroupées en coopératives, amélioration de l'habitat, etc.) permettent aux communautés de développer des activités de conservation de l'environnement, et de diversifier leurs sources de revenus, tout en améliorant leur résilience climatique à travers un spectre plus étendu d'actifs financiers.

- Développement de l'activité touristique. Cette activité permet de générer des fonds utiles à la gestion du paysage et à sa conservation.

- Promotion de la domestication des PFNL (bambous, miel, champignons, etc.) et de l'agroforesterie en milieu rural avoisinant les aires protégées. Ces produits se sont montrés plus résilients face aux variations climatiques.

- Appui à l'élaboration des plans forestiers des districts, facilitant les processus de la REDD+.

\section{Défis et contraintes pour la REDD+ et l'adaptation}

La région est confrontée à une série de menaces qui réduit la résilience climatique des populations et complique la mise en œuvre de la REDD+ :

- L'insuffisance d'encadrement des populations et de renforcement des capacités des gestionnaires du paysage, résultant d'actifs sociaux faibles, qui sont pourtant un facteur important dans les projets d'adaptation. 


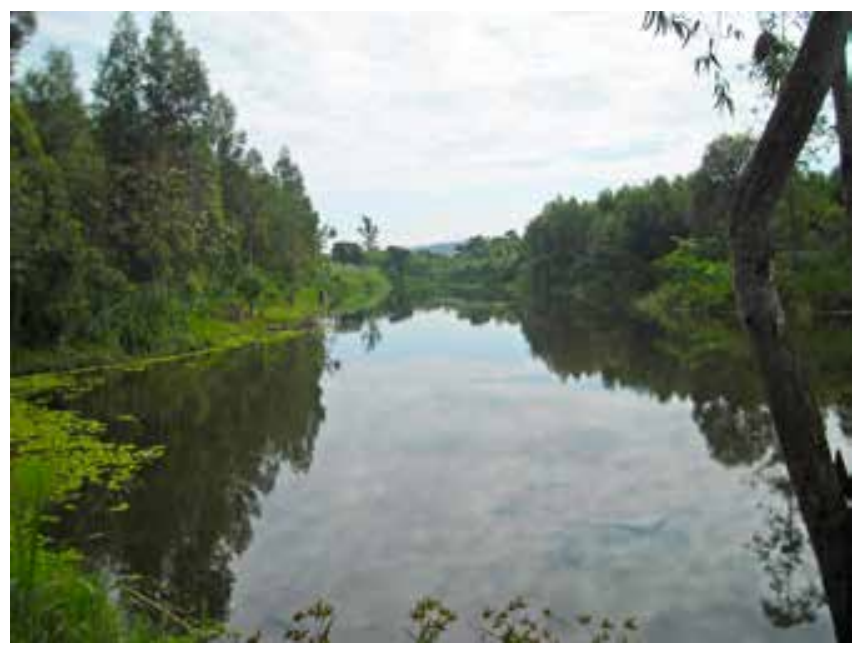

\section{Etang piscicole aménagé pour la pêche de subsistance (Auteur : Claude Sikubwabo)}

- L'invasion du paysage par les populations fuyant la guerre et par les militaires, qui y ont établi des bases et des camps; ceci entraine l'instabilité sociale et l'insécurité dans le paysage et ses environs.

- L'exploitation minière illégale dans les aires protégées, qui contribue à décourager les interventions de la REDD+.

- L'exploitation industrielle de bois non contrôlée.
- $\quad$ L'insuffisance de dispositifs juridiques et de politiques d'adaptation fiables.

- L'insuffisance de moyens humains et financiers pour une meilleure gestion du paysage, afin de contrecarrer la surexploitation des ressources naturelles.

- L'insuffisance d'outils de planification et de gestion durable du paysage.

- La faible participation des communautés dans la gestion des aires protégées, ce qui a un impact sur les structures de gouvernance de la REDD+.

- La pratique de techniques agricoles peu performantes, qui encourage d'un côté l'usage d'aires plus étendues pour des objectifs de rendement, induisant la déforestation, et de l'autre côté, réduit la capacité d'adaptation basée sur les ressources naturelles.

\section{Sources consultées}

Denvers, D. et Vande Weghe, J. P. (coord.) 2007 Les forêts du Bassin du Congo : Etat des forêts 2006. Office des publications de l'Union européenne, Luxembourg.

De Wasseige, C. et Denvers, D. (éd.) 2009 Les forêts du Bassin du Congo : Etat des forêts 2008. Office des publications de I'Union européenne, Luxembourg.

De Wasseige, C. et Denvers, D. (éd.) 2011 Les forêts du Bassin du Congo : Etat des forêts 2010. Office des publications de I'Union européenne, Luxembourg.

Ko, J. 2011 Rapport annuel 2010 : Activités USAID/ CARPE dans les paysages COMIFAC/ PFBC à l'appui du plan de convergence de la COMIFAC (septembre 2009 - septembre 2010).

Wengamulay, J. 2011 Paysage Virunga et le changement climatique. Présentation à l'atelier de Douala, du 12 au 14 mai 2011.

Cette recherche a été menée par le CIFOR dans le cadre du programme de recherche du CGIAR sur les forêts, les arbres et l'agroforesterie. Ce programme collaboratif vise à améliorer la gestion et l'utilisation des forêts, de l'agroforesterie et des ressources génétiques des arbres à l'échelle du paysage, des forêts aux exploitations agricoles. Le CIFOR dirige ce programme collaboratif en partenariat avec Biodiversity International, le CIRAD, le Centre international d'agriculture tropicale et le Centre mondial de l'Agroforestrie.
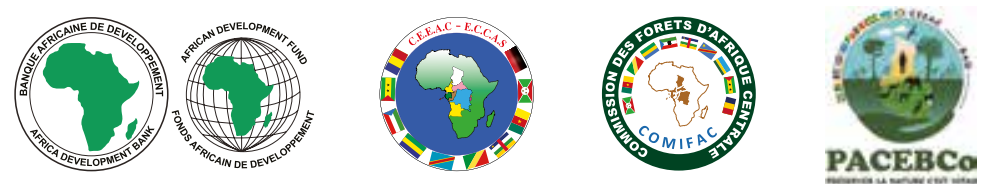

cifor.org/cobam

blog.cifor.org

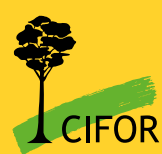

Center for International Forestry Research

CIFOR défend le bien-être humain, la conservation de l'environnement et l'équité en menant une recherche pour éclairer les politiques et les pratiques qui affectent les forêts dans les pays en développement. CIFOR est un centre de recherche du Consortium du CGIAR. Le siège du CIFOR est situé à Bogor, en Indonésie. CIFOR a également des bureaux en Asie, en Afrique et en Amérique du Sud. 